\title{
Deep subwavelength nanometric image reconstruction using Fourier domain optical normalization
}

\author{
Jing Qin, Richard M Silver, Bryan M Barnes, Hui Zhou, Ronald G Dixson and Mark-Alexander Henn
}

Quantitative optical measurements of deep subwavelength, three-dimensional (3D), nanometric structures with sensitivity to sub-nanometer details address a ubiquitous measurement challenge. A Fourier domain normalization approach is used in the Fourier optical imaging code to simulate the full 3D scattered light field of nominally $15 \mathrm{~nm}$-sized structures, accurately replicating the light field as a function of the focus position. Using the full 3D light field, nanometer scale details such as a 2 nm thin conformal oxide and nanometer topography are rigorously fitted for features less than one-thirtieth of the wavelength in size. The densely packed structures are positioned nearly an order of magnitude closer than the conventional Rayleigh resolution limit and can be measured with sub-nanometer parametric uncertainties. This approach enables a practical measurement sensitivity to size variations of only a few atoms in size using a high-throughput optical configuration with broad application in measuring nanometric structures and nanoelectronic devices.

Light: Science \& Applications (2016) 5, e16038; doi:10.1038/lsa.2016.38; published online 26 February 2016

Keywords: computational microscopy, light scattering, metrology, quantitative nanoscale microscopy, sub-nanometer uncertainties

\section{INTRODUCTION}

There have been several recent developments using super-resolution imaging that have shattered the resolution limit using fluorescing techniques $^{1-3}$, pushing image resolution up by two orders of magnitude for certain objects with specific materials properties, e.g., the color centers of diamond ${ }^{4}$. Super-resolved fluorescence microscopy methods have provided significant advances in particle position measurements by localization of the emitting fields yielding sub-nanometer position resolution; however, they have limited use in measuring deep subwavelength detailed geometry ${ }^{2,4-7}$. Near-field scanning optical methods have also demonstrated nanometer sensitivities by operating in the evanescent regime ${ }^{8-10}$ but these methods can be cumbersome to apply relative to far-field optical methodologies. For non-fluorescing materials, other methodologies ${ }^{11,12}$ including optical diffraction tomography (ODT) $)^{13-18}$ have enhanced edge-based imaging such that image resolution has been improved by more than a factor of two ${ }^{12,17}$ beyond conventional optical methods. An alternative approach to access super-resolution information using 'conventional' resolution methods is to use a physical model and limited a priori knowledge ${ }^{19,20}$ to yield deep subwavelength information from the scattered field. However, interpreting the images from diffraction-limited scattered electromagnetic fields of finite, generic three-dimensional (3D) structures well below the conventional Rayleigh resolution limit has remained elusive, primarily since the individual line edges are unresolved at these length scales.

Here, we present an optical microscopy method to comprehensively determine the geometry of features one-thirtieth or smaller than the wavelength of light, using limited a priori information, by addressing limitations in the measurement and normalization of the complex set of amplitude, phase, and spatial frequency propagation errors. Superresolution techniques such as ODT can also be enhanced with a priori information achieving a transverse resolution of $\lambda / 15$ by placing bounds on the permittivity ${ }^{21}$. Other high-throughput model-based methods such as scatterometry and ellipsometry have been widely used recently to measure arrays of lines as small as $10 \mathrm{~nm}$, but these methods are normally used with large repeating arrays of features and typically only the specular reflected light is measured and modeled ${ }^{22-24}$. Low-cost, high-throughput optical measurement of finite, deep subwavelength structures is a critical step forward in optical metrology and has very important implications in the advance of nanotechnology and future widespread nanoelectronics manufacturing.

The methods presented here are of particular importance because they enable the quantitative measurement of structures smaller than $10 \mathrm{~nm}$ in size with sensitivity to sub-nanometer variations. Although rigorous modeling of the scattered electromagnetic field to better estimate edge position or particle position has been successfully used for many years, in microscopy this has been mainly limited to structures that were nominally wavelength sized or larger ${ }^{25,26}$. Recent efforts at the National Institute of Standards and Technology (NIST) have enabled sensitivities to subwavelength features by measuring the $3 \mathrm{D}$ scattered field above the sample using angle-resolved and focusresolved imaging ${ }^{23,27-30}$, known in the literature as scatterfield optical microscopy and through-focus scanning optical microscopy. For example, an empirical differential volumetric (3D) approach for processing through-focus images of sub-20 nm defects has yielded up to fourfold greater defect sensitivities compared to conventional 
two-dimensional (2D) imaging ${ }^{30}$. The through focus imaging method presented here utilizes scattered phase and spatial frequency information captured in focus-resolved images to enable the quantitative reconstruction of finite, subfield $3 \mathrm{D}$ structures as often encountered in nanoelectronics and nanotechnology.

\section{MATERIALS AND METHODS}

Image reconstruction overview

This technique for extracting nanometric quantitative data from scattering data using optical microscopy involves several key technologies summarized here and innovative methodologies that are developed in this section. Imaging the target through multiple focus positions, illustrated in Figure 1a, allows experimental reconstruction of the scattered volume of light. An in-house developed rigorous coupled-

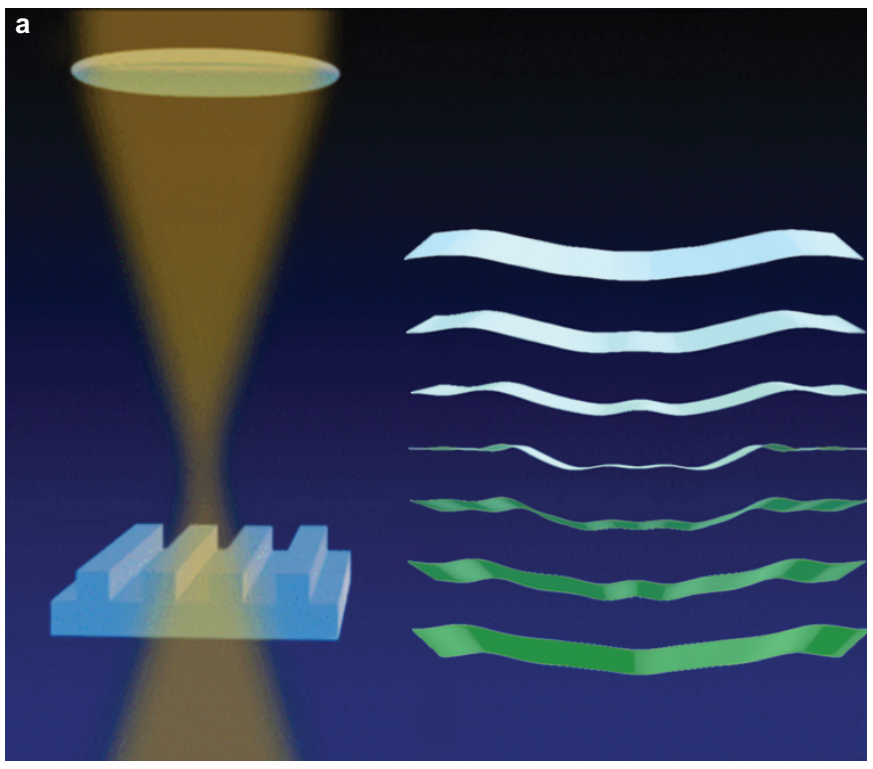

b Simulated images
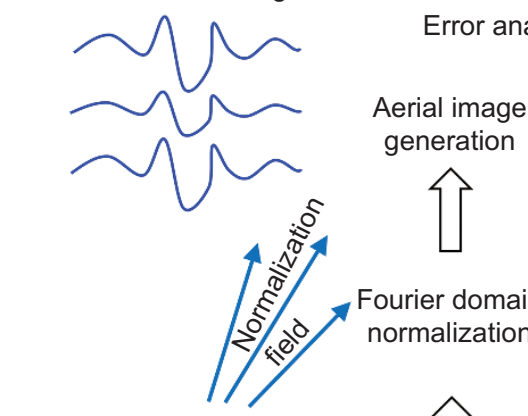

Experimental images

Fourier domain normalization
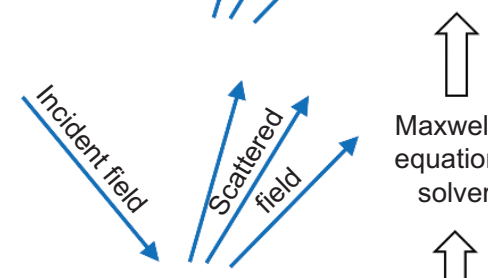

Maxwell's equations solver
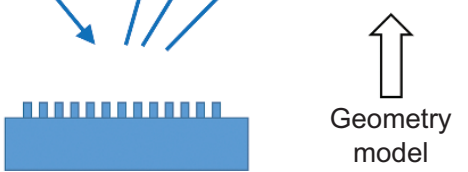

Figure 1 (a) Variation in scattered intensity profile shape with change in focus position. (b) Determination of geometries using solutions to the forward scattering problem with limited a priori knowledge.

Fit experiment

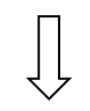

Determine model parameters wave analysis (RCWA) simulation code is used to model the scattered electromagnetic field ${ }^{31}$, with model verification using finite difference time domain (FDTD) ${ }^{32,33}$. Fourier optical methods are used to simulate the illumination path and collection path optics ${ }^{28}$. The method relies on characterizing the optical transfer function of the microscope and 'normalizing' the simulated propagating field through the microscope, enabling the fitting of the 3D experimental intensity measurements by a parametric simulation, illustrated in Figure $1 \mathrm{~b}$.

\section{The hardware platform}

Utilizing these focus-resolved images requires a thorough understanding of the optical paths. Fully characterizing the optical metrology instrument as a function of illumination or scattered spatial frequencies requires full angular control of the light, while the measurement of the targets requires full focus control. Both were performed using an in-house designed microscope, illustrated in Figure 2. A royal blue light-emitting diode (LED), filtered using a nominally Gaussian $\lambda=450 \pm 10 \mathrm{~nm}$ band-pass filter, back-illuminates a holographic diffuser at the microscope source plane. Coherence length can be approximated as $\lambda_{0}^{2} / \Delta \lambda=10 \mu \mathrm{m}$. This diffuser is imaged onto a conjugate to the back focal plane (CBFP). Using Köhler illumination, each point of the CBFP maps to a plane wave at a specified incident angle of illumination. The light is linearly polarized near the CBFP with the transmission axis either parallel or perpendicular to the finite set of features at the sample plane. A single aperture nominally 1200 $\mu \mathrm{m}$ in diameter is positioned in the CBFP, yielding an illumination numerical aperture (INA) of 0.13 . The relatively small INA ensures that the illumination creates a well-defined, narrow cone of illumination. The objective is 0.95 NA with $150 \times$ magnification.

In this work, the microscope is operated in two modes. For the tool characterization, the aperture is rastered in the CBFP to enable angleresolved intensity measurements at the sample plane (using a photodiode) and of reflected light at the image plane (using a charge coupleddevice (CCD) camera) for Fourier domain normalization. For the collection of focus-resolved scattering images from a finite set of features, this aperture in the CBFP is placed on the optical axis. The reflected and captured scattered field is transmitted through a beamsplitter onto a CCD that detects each of the defocused images. It is important to note that each spatial frequency scattered by the finite set of features scatters at a different angle, and various tool imperfections (aberrations of the optical system, transmission losses, etc.) will affect the resulting image as functions of both scattering angle and polarization.

\section{Sample design and reference characterization}

Experiments on sets of targets with nominally 14-, 16-, and 18-nm wide lines were performed such that sets of images were acquired in a focus-resolved mode capturing the 3D-scattered intensities ${ }^{34}$. The specific finite target designs shown in Figure $3 \mathrm{a}$ and $3 \mathrm{~b}$ were fabricated by SEMATECH using e-beam lithography with target areas as small as $1.75 \mu \mathrm{m} \times 6 \mu \mathrm{m}$ (30 lines) and $6 \mu \mathrm{m} \times 6 \mu \mathrm{m}$ (100 lines). These areas compare very favorably with typical scatterometry target sizes of 1600 $\mu \mathrm{m}^{2}$, showing a reduction of more than two orders of magnitude. (Certain commercial equipment, instruments, or materials are identified in this paper to specify the experimental procedure adequately. Such identification is not intended to imply recommendation or endorsement by the NIST, nor is it intended to imply that the materials or equipment identified are necessarily the best available for the purpose.) Line extensions were included to facilitate atomic force microscopy (AFM) measurements that serve as the starting point for 


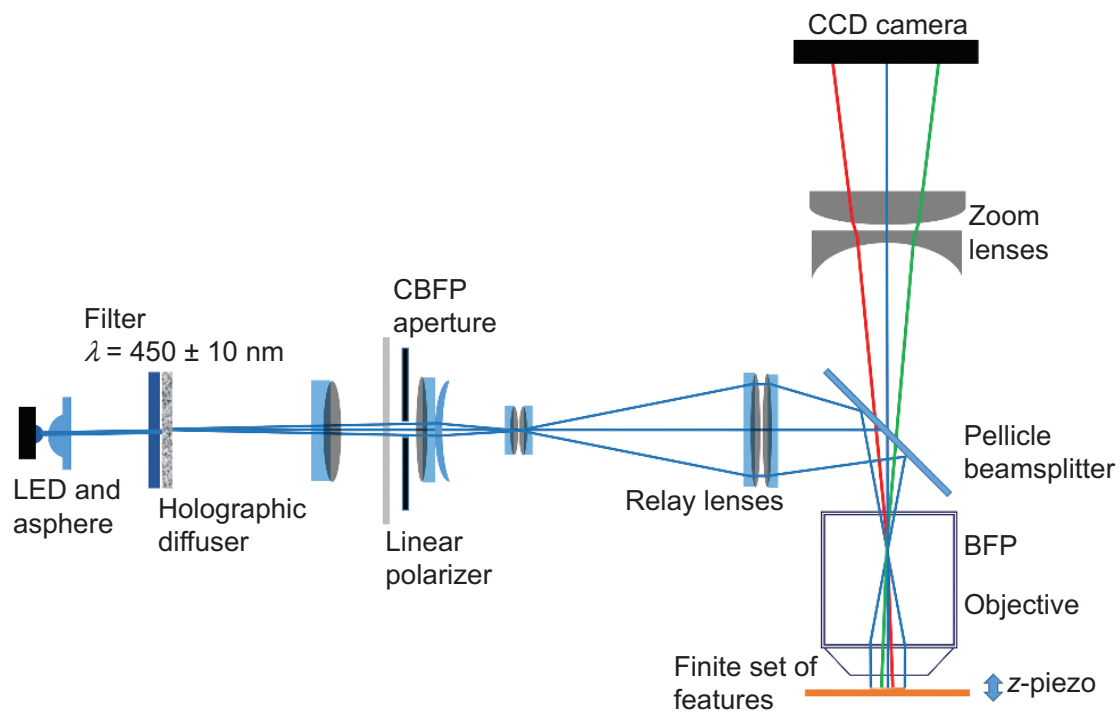

Figure 2 Schematic of the microscope apparatus.

parametric fitting and are also used to independently compare the final results. A transmission electron microscope (TEM) image of a single line from a similar sample indicates a relatively broad base with a narrower width at the top as shown in Figure 3c. Also, for these Si on Si targets, the TEM indicates a thin, native conformal oxide layer 2-3 $\mathrm{nm}$ thick that was included in the parametric model as seen in Figure 3d. Evaluated as a floated parameter in initial fits, this oxide layer is fixed to $2 \mathrm{~nm}$ width at each sidewall with a 3 -nm thick capping layer.

\section{Image corrections and alignment}

A prerequisite to fitting the data was the acquisition and subsequent image subtraction of the dark current of the photodiode and CCD camera and glare. Then, since the targets are finite and the lateral intensity data are used in the analysis, an exhaustive field correction methodology was developed to account for image field intensity inhomogeniety. As data at different focus positions were collected repeatedly, a focus metric ${ }^{27}$ was applied to determine the relative focus positions and enable alignment of the sample plane in the vertical direction. Since the focus metric has $\pm 25 \mathrm{~nm}$ uncertainty, the data-sets were interpolated in $4 \mathrm{~nm}$ intervals for best focus alignment. Lateral positions were also aligned using correlation algorithms, necessary for theory to experiment comparisons during parametric fitting.

\section{Fourier domain normalization}

To implement accurate parametric fitting methods that allow comparison of focus-resolved measurements with electromagnetic scattering simulations, proper experimental normalization procedures combined with rigorous modeling are needed. The microscope consists of two groups of optical elements: illumination path optics and collection path optics. Both paths introduce significant instrumentation errors to the image that must be corrected.

Using Köhler illumination with a finite aperture, the light in the illumination path can be treated as the sum of plane waves from points in the back focal plane of the objective lens. After scattering at a target, however, light propagates through the collection path optics at multiple angles due to multiple scattering frequencies. For finite, very small subimage field targets or non-repetitive, irregular structures that scatter a broad range of continuous frequency content, the normalization required to correct the experimental data for instrumentation and hardware errors becomes complex. An illumination path tool function I and a collection path tool function $\mathbf{C}$ must be calculated and implemented separately for each individual illumination angle
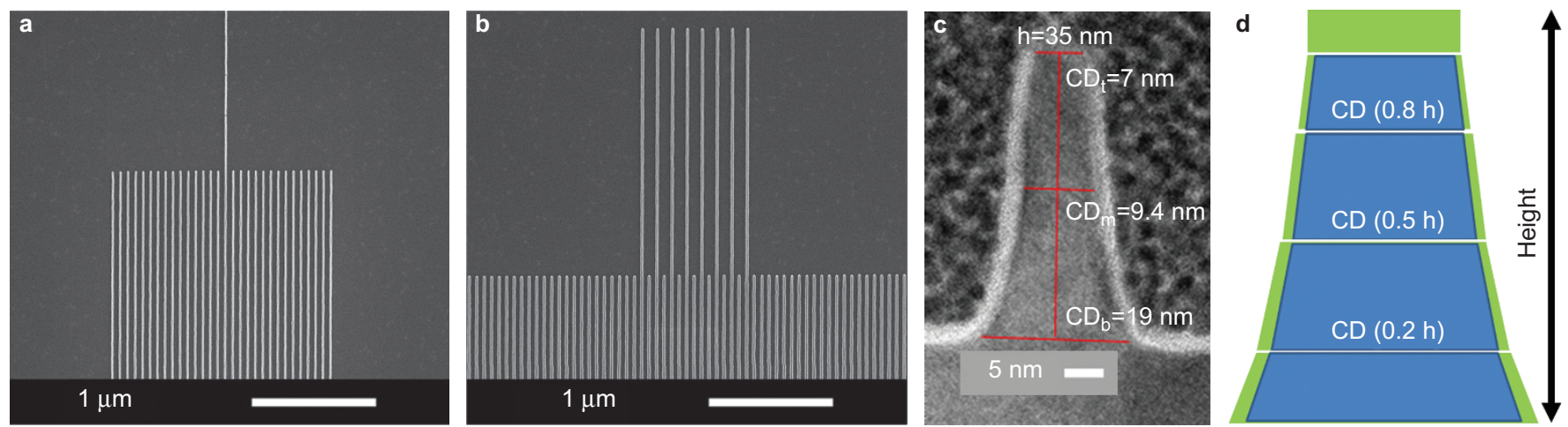

Figure 3 (a) SEM top-down image of 30-line and (b) 100-line targets, from Ref. 28, and (c) TEM cross-section image of a single line profile from a similar sample. (d) Quadruple trapezoid model of line profile with floated parameters (total height and critical dimension (CD) at three heights) for the simulations. 
and the resulting vast spatial frequency spectrum of the scattered light. Although the actual acquisition of the illumination tool function and collection path tool functions have been described elsewhere ${ }^{28}$, a mathematical summary of the resulting matrix implementation is given in the Supplementary Information.

A generalized equation can be constructed in the Jones calculus demonstrating the use of these tool functions on individual components of the scattered field resulting from a source plane wave ${ }^{35}$. The scattered field can be discretized into a set of Fourier components, indexed by $i, j$ that yields for each individual component:

$$
\boldsymbol{U}_{i j}=\mathbf{C}_{i j} \cdot \mathbf{S}_{i j} \cdot \mathbf{I} \cdot \boldsymbol{U}_{0}
$$

where $\boldsymbol{U}_{0}$ and $\boldsymbol{U}_{i j}$ are Jones vectors with $\boldsymbol{U}_{0}$ representing the amplitude of the source plane wave and $\boldsymbol{U}_{i j}$ the amplitude of the $i j^{\text {th }}$ Fourier component of the scattered plane wave, $\mathbf{I}$ is the Jones matrix for the illumination tool function, and $\mathbf{C}_{i j}$ and $\mathbf{S}_{i j}$ are Jones matrices for the collection tool function and the scattering, respectively, for the $i j^{\text {th }}$ component. In this formulation, the specific polarization bases have yet to be specified nor are any required rotational matrices included. Here, the source is linearly polarized either along the $x$ or $y$ axes in the CBFP, axes which are aligned to the finite set of features and $U_{\boldsymbol{0}}$ is either $\left[\begin{array}{l}1 \\ 0\end{array}\right]$ or $\left[\begin{array}{l}0 \\ 1\end{array}\right]$ for $x$ or $y$ polarizations, respectively. The experimental determination of the $\mathbf{I}$ and $\mathbf{C}$ matrices and our particular implementation of Equation (1) are discussed in the Supplementary Information.

With a complete knowledge of normalization functions I and C, complex samples can be measured by assuming a particular geometry for which the electromagnetic scattering $S_{i j}$ can be numerically calculated as a function of a parametric geometrical model constructed as input for a numerical Maxwell's equations solver with nanoscale resolution. Less a priori information could have been used at a greater computational cost, for example, optical constants of the features and substrate could have been floated as well as the period. Judicious use of a priori knowledge of some of these characteristics is very useful to maximize information from the scattered signal in determining unknown characteristics. Moreover, visualizing edge positions to determine feature width or spacing is not required when comparing the forward scattering from a parametric model to the measured intensity.

To solve the inverse problem, a multidimensional library of these simulations, indexed by the parameters defining that geometry, is generated using the RCWA by solving Maxwell's equations numerically using in-house developed code. As RCWA is inherently periodic, a larger periodic domain containing the finite structure is used to approximate optical isolation of the structure. Convergence testing was performed to determine the appropriate size of the domain required to minimize optical interactions below a set threshold. Inhouse model development is preferable as Fourier domain normalization is performed on the accessible Fourier scattering components that are calculated through simulation of the scattered electromagnetic field $\vec{E}_{\text {scat }, m}(x, y)$, using:

$$
\vec{F}_{\text {scat }, m}\left(k_{x}, k_{y}\right)=\iint_{-\infty}^{\infty} \vec{E}_{\text {scat }, m}(x, y) \cdot e^{-i 2 \pi\left(k_{x} x+k_{y} y\right)} \mathrm{d} x \mathrm{~d} y
$$

where the subscript 'scat, $m$ ' denotes the scattered field for a given linear polarization basis ('pol') selected at the CBFP for the $m^{\text {th }}$ individual source plane wave defined by its polar and azimuthal angles of incidence, $\theta_{m}$ and $\phi_{m}$. It is from successive calculations of this Fourier vector transform $\vec{F}_{\text {scat }, m}\left(k_{x}, k_{y}\right)$ for the two orthogonal polarizations that the $\boldsymbol{S}_{i j}$ matrix is determined. Illumination and collection path normalization is accomplished by applying scalar corrections in the Fourier domain, using the illumination and collection tool functions, to each of the scattered frequency components $\vec{F}_{\text {scat }, m}\left(k_{i}, k_{j}\right)$ with:

$$
\vec{F}_{\text {scat }, m}^{\prime}\left(k_{x}, k_{y}\right)=C_{\text {pol }}\left(k_{x}, k_{y}\right) \vec{F}_{\text {scat }, m}\left(k_{x}, k_{y}\right) I_{\text {scat }, m}
$$

where $C_{\mathrm{pol}^{\prime}}$ is also dependent on the polarization of the scattered component. These corrected scattered Fourier components are then combined using an inverse Fourier transform:

$$
\vec{E}_{\text {scat }, m}^{\prime}(x, y)=\int_{-\infty}^{\infty} \int_{F_{\text {scat }, m}^{\prime}}\left(k_{x}, k_{y}\right) \cdot e^{i 2 \pi\left(x k_{x}+y k_{y}\right)} \mathrm{d} k_{x} \mathrm{~d} k_{y}
$$

to compute the normalized field near the image plane.

The low coherence length of the LED is well approximated by treating the source as incoherent. Therefore, with a finite aperture and Kohler illumination, multiple plane waves are simulated resulting in a normalized $\vec{E}_{\text {scat,m }}^{\prime}$ for each of these. The normalized image for each incident polarization is then calculated using:

$$
I_{\text {pol }}(x, y)=\sum_{m=1}^{n}\left|\vec{E}_{\text {scat }, m}^{\prime}(x, y)\right|^{2}
$$

where $n$ is the total number of plane waves simulated. To calculate the images at different focus positions, the normalized fields $\vec{E}_{\text {scat }, m}^{\prime}$ are propagated in $z$ (focus position) using standard methods, the free space Helmholtz equation in the Fourier domain. Two sets of images are then generated as a function of focus position, one set for each polarization.

\section{Regression model}

A nonlinear regression model ${ }^{36,37}$ is used to conduct an accurate and quantitative parametric fit between the measured data and a multidimensional indexed parametric library. An overview to the initial derivation is given here, see Refs. 38,39 for a more detailed formalism.

Minimization is first attempted using particle swarm optimiza$\operatorname{tion}^{40}$. Once a minimum is found, a linear approximation of the nonlinear regression can be made for $y_{i}$, given by

$$
y_{i}=y\left(x_{i} ; \mathbf{a}(0)\right)+\sum_{k=1}^{K}\left[\frac{\partial y\left(x_{i} ; \mathbf{a}\right)}{\partial a_{k}}\right]_{\mathbf{a}=\mathbf{a}(0)}\left(a_{k}-a_{k}(0)\right)+\varepsilon_{i}
$$

where $\mathbf{a}(0)=\left\{a_{1}(0), \ldots, a_{K}(0)\right\}$ and $y_{i}$ is the $i^{\text {th }}$ experimental observation, $\mathbf{a}=\left\{a_{1}, \ldots, a_{K}\right\}$ is a vector of parameters that yield simulated data $y\left(x_{i} ; \mathbf{a}(0)\right)$, and $\varepsilon_{i}$ is the corresponding total error between the experimental value and the best fit simulated value. Expressed in matrix form, $\boldsymbol{\beta}(0)=\boldsymbol{a}-\boldsymbol{a}(0)$, whose generalized least squares estimator is given by:

$$
\hat{\boldsymbol{\beta}}(0)=\left(\mathbf{D}(0)^{\mathrm{T}} \mathbf{V}^{-1} \mathbf{D}(0)\right)^{-1} \mathbf{D}(0)^{\mathrm{T}} \mathbf{V}^{-1} \mathbf{Y}(0)
$$

where $\mathbf{D}$ is a matrix of partial derivatives of the simulated intensities with respect to the components of $\mathbf{a}$, and $\mathbf{V}$ is based upon experimental and estimated uncertainties. The matrix $\mathbf{V}$ need not be a diagonal matrix, and correlations in the estimated phase error are treated with off-diagonal elements. Solving Equation (7) yields 
$\hat{\boldsymbol{\beta}}(0)=\left(\hat{\beta}_{1}(0), \ldots, \hat{\beta}_{K}(0)\right)$, the best linear unbiased estimators of $\boldsymbol{\beta}(0)$. Additional calculation yields the variance of $\boldsymbol{\beta}(0)$, complementing the parametric fit of the structure with uncertainty analysis.

\section{Intensity error analysis}

Accurate evaluation of tool-introduced measurement errors is essential to enable accurate parametric fitting. Here, we developed a new 3D error envelope method to characterize several key systematic errors such as CCD lateral pixel pitch errors, illumination aperture size uncertainty, aperture positioning uncertainty, through-focus stage positioning uncertainty, tool function repeatability errors, and modeling/parameterization errors. Known sources of error from the model can be included as well, e.g., uncertainty from $\mathrm{SiO}_{2}$ thickness. Significant, correlated, but difficult to measure tool errors also include the potential phase errors that result as each diffracted order transmits the microscope optical path as well as random phase errors. These error sources are assumed to be independent and are estimated using simulation, known microscope design aberration, and experimental characterization. The combined set of these components as functions of image pixel and focus position form the new 3D error envelope. These components are assembled to form the $\mathbf{V}$ matrix in Equation (7).
As an example, simulation data are reprocessed 1000 times using the nominal value of the collection numerical aperture, CNA $=0.95$, while adding to this a Gaussian random component $\sigma=0.1$ with an effective upper bound of $\mathrm{CNA}=1$. This randomization generates a set of intensity profiles based on this variation of the CNA, and a covariance matrix $\mathbf{V}_{\mathrm{CNA}}$ is calculated using the 1000 simulations. This process is repeated for each systematic error component and it is through the addition of these matrices, generated through Monte Carlo simulation, that a full $\mathbf{V}$ matrix with potentially off-diagonal elements is determined $^{41}$.

The simplest method for comparing the relative magnitude of the errors is to inspect the diagonal components of the matrix V. In Figure $4 \mathrm{a}$, these values are shown for the 30 -line target at the middle $z$ positon for the $y$ CBFP polarization. Five dominant components are shown: aperture positioning uncertainty (in the $k_{x y}$ space), random phase errors, the collection numerical aperture, through-focus stage positioning uncertainty for polarization $y$, and correlated phase errors. In Figure $4 \mathrm{~b}$, the $2 \sigma$ (two standard deviations) diagonal components of the complete error envelope are shown in red for a 100-line target. For comparison, the $2 \sigma$ experimental repeatability errors are plotted in green and the residuals between best fit and measured data shown in blue. The estimated error envelope shows that the largest errors are at
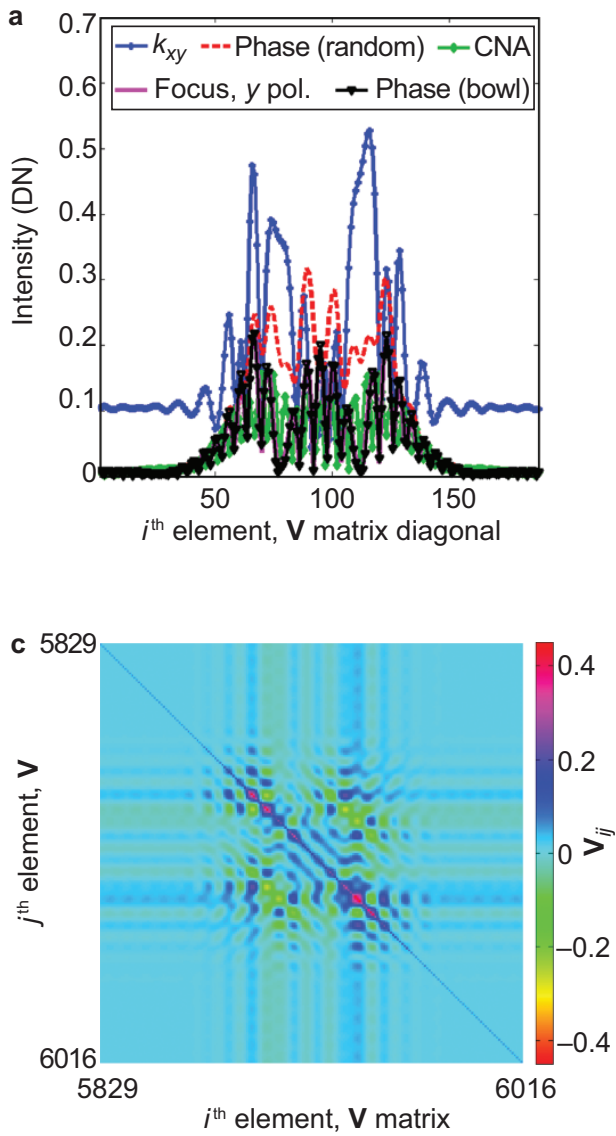
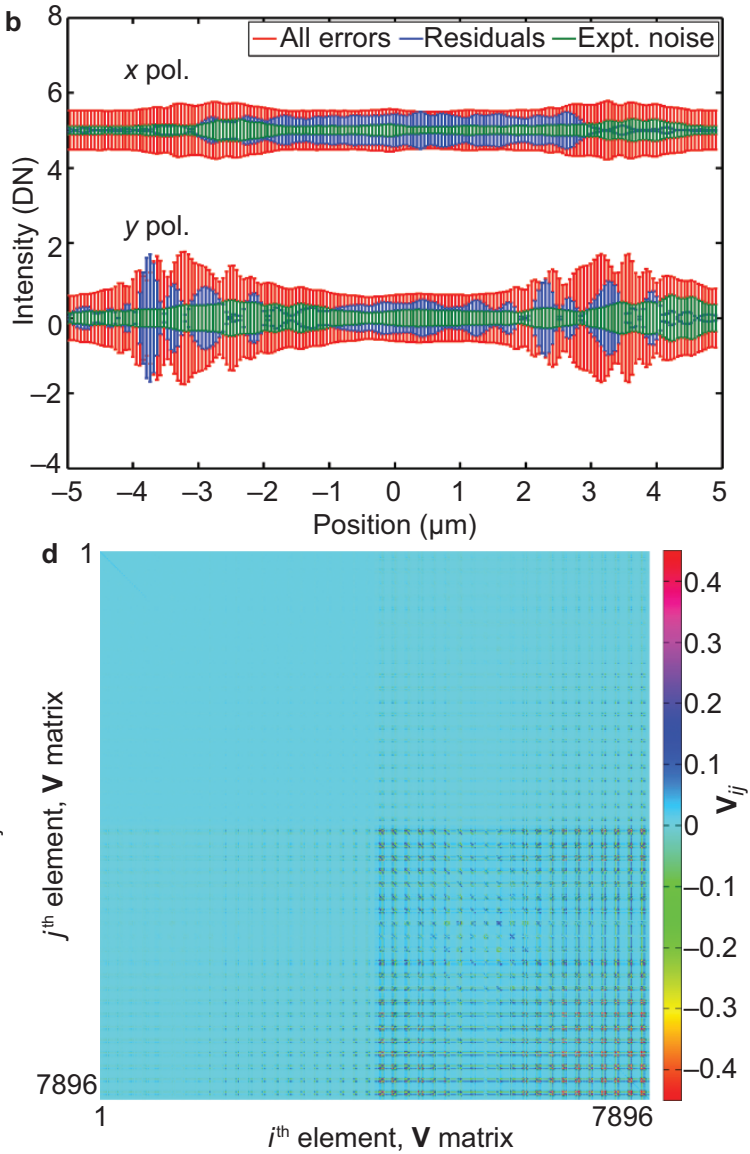

Figure 4 (a) Five significant diagonal elements of the $\mathbf{V}$ matrix for the 30-line target, middle $z$ position, $y$ polarization, $1 \sigma$ (one standard deviation). Shown are the potential errors due to misalignment of the aperture in $k_{x y}$ space, random phase errors, the collection numerical aperture (CNA) error, the focus misalignment for the $y$ polarization, and the correlated phase error that is assumed to be bowl-shaped. (b) $2 \sigma$ error envelopes as functions of polarization for the microscope at one focus position for the 100-line target. Twenty-one error envelopes are calculated since there is a unique error envelope at each focus position. DN is the digital number, the integer readout of the CCD. (c and $\mathbf{d}$ ) Full elements of the $\mathbf{V}$ matrix for the 30-line target for (c) the middle $z$ position, $y$ polarization and (d) the concatenated set of all collected data. The largest correlations fall within the $y$ polarization data-set (lower right quadrant). Color bars correspond to the measured intensity data in DN. 
the edges of the finite arrays, consistent with the residuals. Coverage of the residual error map (blue) by the $2 \sigma$ total error envelope (red) is within the appropriate statistical limits.

How each of these intensity error elements contribute to the final parametric uncertainty is a nonlinear problem due to correlations among the pixels within a single image and also correlations among pixels in the various $z$ planes. Shown in Figure $4 \mathrm{c}$ is the subset of the full V matrix for a 30-line target with all components added together that corresponds to the middle $z$ position of the $y$ polarization. The entire $\mathbf{V}$ matrix is shown in Figure 4d for comparison. The full 3D error envelope is realized by concatenating each $z$ position and polarization together to form this full $\mathbf{V}$ matrix.

\section{RESULTS AND DISCUSSION}

The methods are applied here to a fundamental transition regime in optical scattering, the scattering from an optically finite set of features. In one limit, conventional scatterometry arrays are designed such that the optical spot size is smaller than the array, allowing an approximation of the array as infinitely periodic ${ }^{42}$. This estimate yields discrete diffraction orders governed by the grating equation. In the other limit ${ }^{28}$ scattering from a single edge was measured and fit using parametric optical modeling of the continuum of frequencies scattered from an isolated single edge. Between these two limits lies the broad region of finite and periodic structures, the critical region measured here. The method presented here is broadly applicable to this region. We explore in detail the situation where several similar structures are grouped together because this has the effect of significantly enhancing sub-nanometer sensitivity and is likely to provide a very useful target design widely applicable in several forms of nanoelectronics manufacturing.

Figure 5 shows results for two 100-line targets with two different line widths at the mid-height of the lines. Here, fitting results from 21 different focus positions are plotted, offset in intensity for clarity. Figure $5 \mathrm{a}$ and $5 \mathrm{~b}$ corresponds to the nominally $14 \mathrm{~nm}$ wide lines, and Figure $5 \mathrm{c}$ and $5 \mathrm{~d}$, the nominally $16 \mathrm{~nm}$ lines. (Note that these feature widths are nominally $1 / 28^{\text {th }}$ the wavelength.) The normalized simulation-to-experimental profile comparisons show good agreement throughout the entire $4 \mu \mathrm{m}$ focus range. Only the diagonal elements of the $\mathbf{V}$ matrix in the intensity error analysis were applied to these data, and some residuals localized at the array edges ocassionally exceed the $1 \sigma$ error bars and are indicative of uncorrected, correlated systematic error.

In simple terms, the middle portion of the 100-line array for this set of dimensions and measurement wavelength acts as a specular optical grating, and little optical variation is observed in the middle region going through focus. The two edges therefore are optically isolated. Another target was designed to enhance the interaction between the two edges of the arrayed set of features. Figure 6 shows fitting examples for a 30-line target with nominally $14 \mathrm{~nm}$ mid-height linewidth and top-width of $10 \mathrm{~nm}$, nominally one-fortieth the wavelength. For these data, the full $\mathbf{V}$ matrix from the intensity error analysis have been applied prior to the regression. It is interesting to examine the complex scattered field resulting primarily from the first 10-20 lines at the array edge. This region of the scattered field results primarily from several subwavelength features scattering in an intermediate domain between grating and isolated feature behavior and contains a wealth of optical information with respect to phase, amplitude and frequency content. The best fit parameters a (Equation (6)) and the uncertainty analysis from linear regression are shown in Table 1.

The relative size of the parametric uncertainties in a is remarkably small and requires discussion. The parametric uncertainties only represent the expanded measurement uncertainty ${ }^{43}$ if all contributions to uncertainty have been accounted for in the $\varepsilon_{i}$ of Equation (6) and the appropriate corresponding parameters have been floated. Ultimately, the uncertainty is fundamentally limited by the $\varepsilon_{i}$ and the correlation among floated parameters. To obtain the best achievable uncertainty, it is of paramount importance that the residual errors in the final fit are statistically covered by the uncertainty envelope and the optimum number of parameters are floated to balance the corresponding increase in uncertainty, the computational costs due to the increased
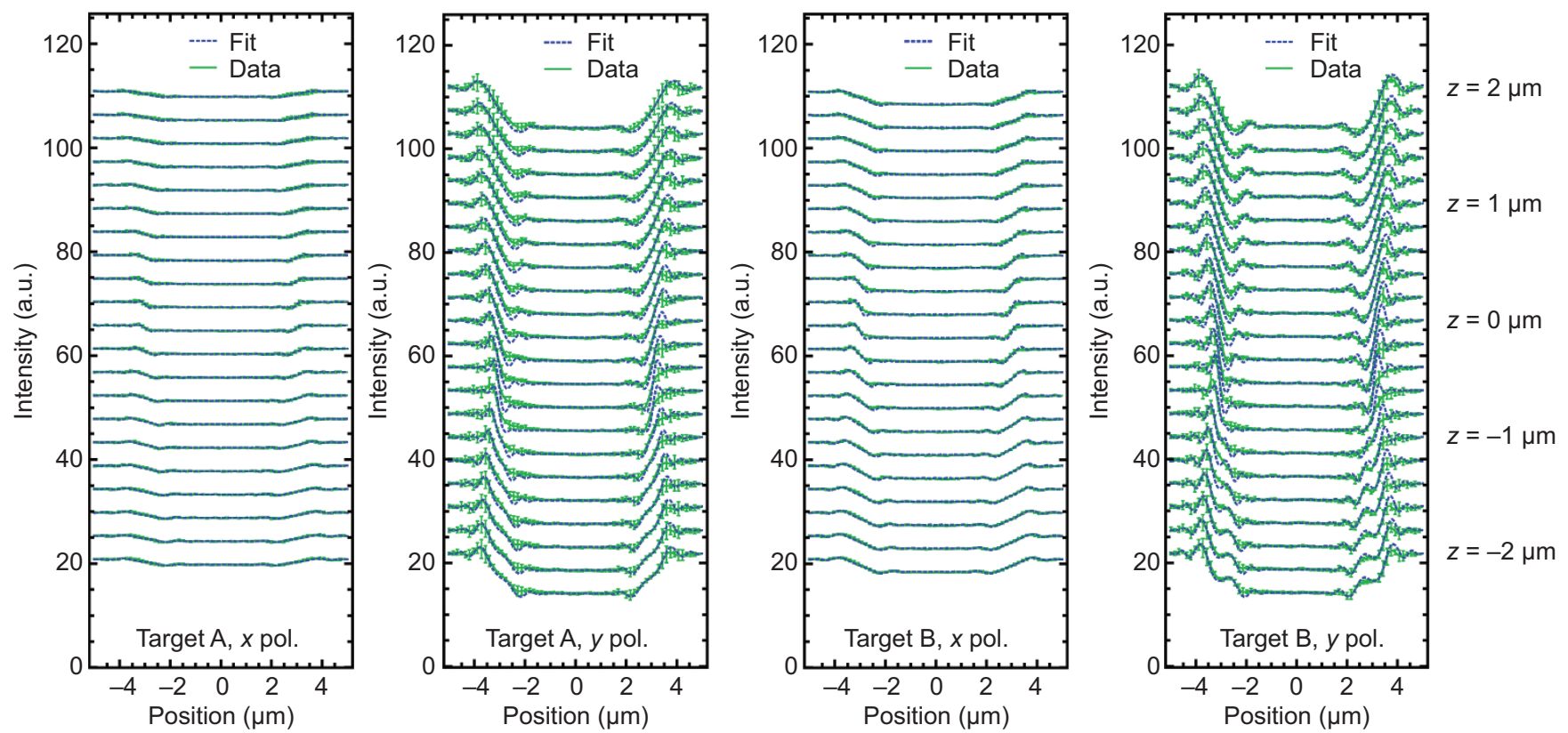

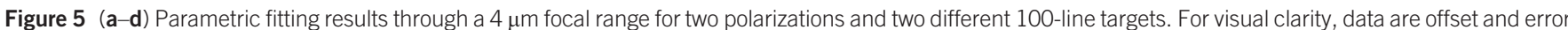
bars are staggered. 

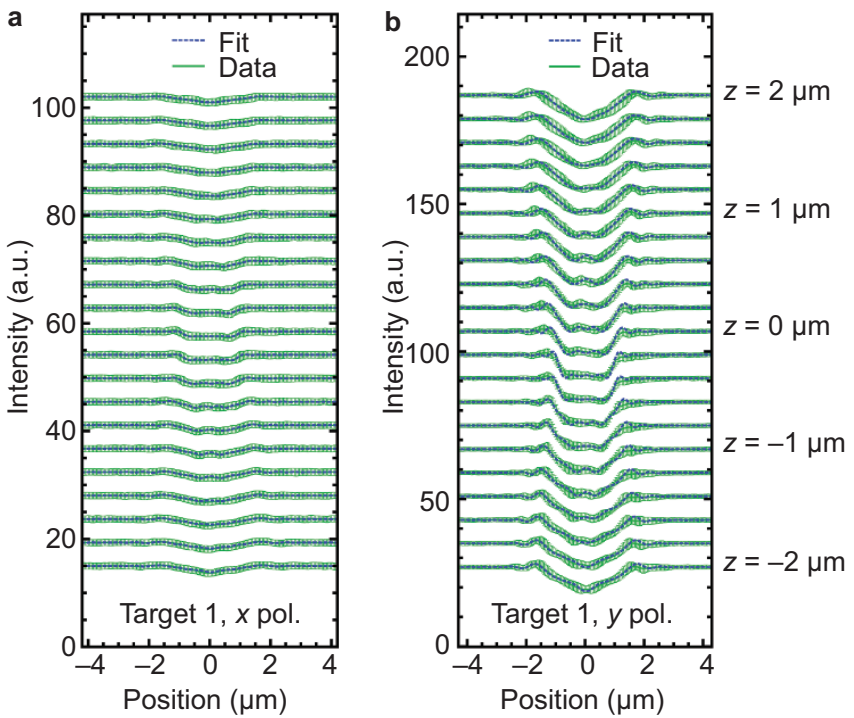

Figure 6 Parametric fitting results of a 30-line target through the entire $4 \mu \mathrm{m}$ focus range for two polarizations. For visual clarity, data are offset.

number of 3D simulations, and the magnitude of the residuals. A more detailed discussion of the statistical analysis presented here and complexities endemic to nonlinear regression fitting is beyond the scope of this paper ${ }^{39}$. The best recommendation for fitting such data involves floating an increasing number of parameters and obtaining the appropriate reduced chi-squared value. In the examples shown here, the basic methodology of fitting finite, nanometric optical targets is demonstrated with four-parameter data sets.

For each of these parameter fits, the uncertainties were sampled within a region $2 \mathrm{~nm}$ wide in each of the four parametric dimensions. The uncertainties in Table 1 are averages of these uncertainties, obtained from linear approximations of the nonlinear regression.

In Figure 7, correlations between optical CD (OCD) data using this technique and AFM reference data are shown. Here, parametric fitting results of the 'top' and middle line widths for the 30-line targets are plotted versus the corresponding AFM measurement data. While both the AFM and OCD middle widths are measured at half the line height $(0.5 \mathrm{~h})$, the OCD and AFM have different but related top width measurands as the AFM is reported at $0.95 \mathrm{~h}$, the OCD at $1.0 \mathrm{~h}$. The fittings are linear functions with an offset between the top and middle widths. Comparisons were also made with rigorous Monte Carlo-based scanning electron microscope (SEM) measurements and an approximate 0-4 $\mathrm{nm}$ bias is present and under investigation, but it must be noted

Table 1 Chi-squared best fits of parameters and parametric uncertainties $\sigma_{\beta}(k=2)$ from linear regression of the image profiles from 30-line targets as shown in Figure 6.

\begin{tabular}{|c|c|c|c|c|c|c|}
\hline & \multicolumn{2}{|c|}{ Target 1} & \multicolumn{2}{|c|}{ Target 2} & \multicolumn{2}{|c|}{ Target 3} \\
\hline & a & $\sigma_{\beta}$ & a & $\sigma_{\beta}$ & a & $\sigma_{\beta}$ \\
\hline Height (nm) & 34.1 & 0.7 & 34.7 & 0.5 & 36.1 & 0.6 \\
\hline CD $(1.0$ h) (nm) & 14.5 & & 22.7 & & 27.4 & \\
\hline$(0.8$ h) $(\mathrm{nm})$ & 16.5 & 2.2 & 24.7 & 2.1 & 29.4 & 4.4 \\
\hline$(0.5$ h) (nm) & 18.2 & 0.7 & 23.7 & 0.8 & 28.5 & 1.3 \\
\hline$(0.2 \mathrm{~h})(\mathrm{nm})$ & 18.2 & 0.8 & 22.7 & 1.0 & 27.5 & 1.2 \\
\hline$(0.0$ h) (nm) & 22.2 & & 26.7 & & 31.5 & \\
\hline
\end{tabular}

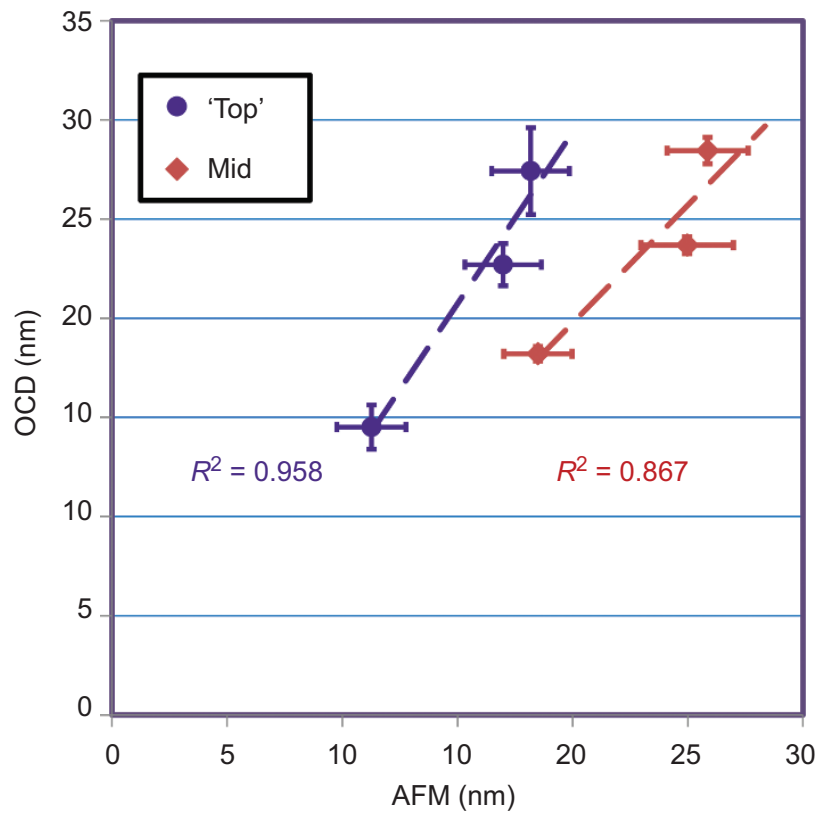

Figure 7 Correlation between optical CD data and AFM reference data (Pearson $r$ ). Error bars are $1 \sigma(k=1)$.

that there are actual differences in the measurands, since the SEM measurements are an average of local measurements and not the larger average array values; similarly, the AFM measures the single line extensions and the optical technique measures the primary array. In addition, isolated patterns tend to etch differently relative to more dense patterns, and the optical method is less sensitive to sidewall oxide than the AFM.

This limited set of similar features improves sensitivity so dramatically because of the strong imaging dependence on the interacting scattered phase, frequency, and amplitude. This enhanced scattering is a combination of edge effects at the extents of the array and also the presence of nonspecular scattering from interior array scatterers that would otherwise be dampened for an infinite array given the same feature geometry. The technique is most effective using novel finite structures with pitch less than one-tenth the illumination wavelength and simulation results indicate that feature widths can be one-fortieth the wavelength and potentially smaller. The method is so powerful because of the much richer optical information used in the analysis. The key to accurate and quantitative fitting is the application of Fourier normalization of fields modeled using Maxwell's equations field solvers at the nm scale, before image reconstruction combined with a comprehensive error propagation and multidimensional regression of the measured images.

\section{CONCLUSIONS}

In summary, a new optical approach to measure deep subwavelength features with sub-nanometer parametric uncertainties was demonstrated that could have wide-ranging implications for nanotechnology. Similarly, this method could have a major effect on semiconductor manufacturing metrology and process control. Using new advances in accurate optical tool characterization, Fourier domain normalization, and image reconstruction, the method was shown to quantitatively measure finite, nanometer scale targets that scatter a broad range of spatial frequency content. The scattered electromagnetic field was shown to contain a wealth of accessible optical information by modeling the phase, amplitude, and spatial frequency 
through the use of Fourier-based image reconstruction. The approach uses a novel correction for both the illumination and collection path throughout the angular spectrum as a function of polarization. This paper demonstrates that high throughput, nanometric measurements can be achieved using focus-resolved scatterfield microscopy combined with a rigorous regression analysis against a library of physicsbased simulations. A new uncertainty analysis using nonlinear regression and a fundamental advance in the associated error propagation was used to demonstrate sub-nanometer uncertainties. The methodology should be easily extensible to super-resolution techniques such as ODT for which the amplitude and phase are measured. This computational optical approach represents a realistic solution to the very challenging problem of non-destructive measurement of nanometer scale structures with sub-nanometer sensitivity while still having high throughput. With this approach, nanometer scale details such as a thin conformal oxide $2 \mathrm{~nm}$ thick and nanometer topography are rigorously fit with $3 \mathrm{D}$ contours of features as small as $15 \mathrm{~nm}$ in size using $450 \mathrm{~nm}$ wavelength light. The rigorous, quantitative measurement of nanometer scale details of a deep subwavelength, sub-field of view target is a fundamental advance in the measurement of irregularshaped nanometer structures encountered throughout nanoscale science and future nanotechnology products.

\section{AUTHORS' CONTRIBUTIONS}

J.Q., H.Z., B.B., and R.S. designed the experiments. J.Q. and B.B. designed the targets. J.Q. performed the experiments and performed simulations. H.Z. and M.H. developed data fitting and uncertainty calculation methods. R.D. performed the AFM measurements. J.Q., H.Z., B.B., and R.S. interpreted the data, discussed the results, and wrote the paper.

\section{ACKNOWLEDGEMENTS}

The authors would like to thank SEMATECH and Abraham Arceo for fabricating our target designs and providing the TEM micrograph shown as Figure $3 \mathrm{c}$. The authors also wish to thank our colleagues András Vladár and Bin Ming for acquiring and processing the SEM micrographs shown as Figure 3a and $3 \mathrm{~b}$.

1 Hell SW, Wichman J. Breaking the diffraction resolution limit by stimulated emission: stimulated-emission-depletion fluorescence microscopy. Opt Lett 1994; 19: 780-782.

2 Betzig E, Patterson GH, Sougrat R, Lindwasser OW, Olenych S et al. Imaging intracellular fluorescent proteins at nanometer resolution. Science 2006; 313: 1642-1645.

3 Hell SW. Toward fluorescence nanoscopy. Nat Biotechnol 2003; 21: 1347-1355.

4 Rittweger E, Han KY, Irvine SE, Eggeling C, Hell SW. STED microscopy reveals crystal colour centres with nanometric resolution. Nat Photon 2009; 3: 144-147.

5 Mlodzianoski MJ, Juette MF, Beane GL, Bewersdorf J. Experimental characterization of $3 \mathrm{D}$ localization techniques for particle-tracking and super-resolution microscopy. Opt Expr 2009; 17: 8264-8277.

6 Speidel M, Jonáš A, Florin EL. Three-dimensional tracking of fluorescent nanoparticles with subnanometer precision by use of off-focus imaging. Opt Lett 2003; 28: 69-71.

7 Schermelleh L, Heintzmann R, Leonhardt $\mathrm{H}$. A guide to super-resolution fluorescence microscopy. J Cell Biol 2010; 190: 165-175.

8 Kawata S, Inouye Y, Verma P. Plasmonics for near-field nano-imaging and superlensing. Nat Photon 2009; 3: 388-394.

9 Rang M, Jones AC, Zhou F, Li ZY, Wiley BJ et al. Optical near-field mapping of plasmonic nanoprisms. Nano Lett 2008; 8: 3357-3363.

10 Qin J, Iwami K, Umeda N. Direct evaluation of anchoring effects and vertical orientation profiling of liquid crystal films by near-field birefringence measurement. App Phys Expr 2008; 1:111501.

11 Zhou R, Edwards C, Arbabi A, Popescu G, Goddard LL. Detecting 20 nm wide defects in large area nanopatterns using optical interferometric microscopy. Nano Lett 2013; 13: 3716-3721.

12 Rogers ETF, Lindberg J, Roy T, Savo S, Chad JE et al. A super-oscillatory lens optical microscope for subwavelength imaging. Nat Mater 2012; 11: 432-435.
13 Lauer V. New approach to optical diffraction tomography yielding a vector equation of diffraction tomography and a novel tomographic microscope. J Microsc 2002; 205: 165-176.

14 Belkebir K, Chaumet PC, Sentenac A Influence of multiple scattering on threedimensional imaging with optical diffraction tomography. J Opt Soc Am A Opt Image Sci Vis 2006; 23: 586-595.

15 Maire G, Drsek F, Girard J, Giovannini H, Talneau A et al. Experimental demonstration of quantitative imaging beyond Abbe's limit with optical diffraction tomography. Phys Rev Lett 2009; 102: 213905.

16 Girard J, Maire G, Giovannini H, Talneau A, Belkebir K et al. Nanometric resolution using far-field optical tomographic microscopy in the multiple scattering regime. Phys Rev A 2010; 82: 061801.

17 Zhang T, Ruan Y, Maire G, Sentenac D, Talneau A et al. Full-polarized tomographic diffraction microscopy achieves a resolution about one-fourth of the wavelength. Phys Rev Lett 2013; 111: 243904.

18 Arhab S, Soriano G, Ruan Y, Maire G, Talneau A et al. Nanometric resolution with farfield optical profilometry. Phys Rev Lett 2013; 111: 053902

19 Toraldo di Francia G. Resolving power and information. J Opt Soc Am 1955; 45 . 497-499.

20 Danuser G. Super-resolution microscopy using normal flow decoding and geometric constraints. J Microsc 2001; 204: 136-149.

21 Chaumet PC, Sentenac A, Belkebir K, Maire G, Giovannini H. Improving the resolution of grating-assisted optical diffraction tomography using a priori information in the reconstruction procedure. J Modern Opt 2010; 57: 798-808.

22 Huang HT, Terry Jr FL. Spectroscopic ellipsometry and reflectometry from gratings (Scatterometry) for critical dimension measurement and in situ, real-time process monitoring [published erratum appears in Thin Solid Films 2004; 468: 339-346.] Thin Solid Films 2004; 455-456: 828-836.

23 Silver RM, Barnes BM, Attota R, Jun J, Stocker M et al. Scatterfield microscopy for extending the limits of image-based optical metrology. App/ Opt 2007; 46: 4248-4257.

24 Vedam K, McMarr PJ, Narayan J. Nondestructive depth profiling by spectroscopic ellipsometry. App/ Phys Lett 1985; 47: 339-341.

25 Marx E. Images of strips on and trenches in substrates. Appl Opt 2007; 46: 5571-5587.

26 Germer TA, Marx E. Simulations of optical microscope images. In: Archie $\mathrm{CN}$, editor. Metrology, Inspection, and Process Control for Microlithography XX; 19-24 February 2006; San Jose, CA, USA. SPIE: Bellingham, WA, USA, 2006. p615201.

27 Silver RM, Attota R, Stocker MT, Bishop M, Howard L et al. High-resolution optical metrology. In: Silver R, editor. Metrology, Inspection, and Process Control for Microlithography XIX; February 27-March 4 2005; San Jose, CA, USA. SPIE: Bellingham, WA, USA, 2005. p67.

28 Qin J, Silver RM, Barnes BM, Zhou H, Goasmat F. Fourier domain optical tool normalization for quantitative parametric image reconstruction. Appl Opt 2013; 52: 6512-6522.

29 Attota R, Dixson RG. Resolving three-dimensional shape of sub-50 $\mathrm{nm}$ wide lines with nanometer-scale sensitivity using conventional optical microscopes. App/ Phys Lett 2014; 105: 043101.

30 Barnes BM, Sohn MY, Goasmat F, Zhou H, Vladár AE et al. Three-dimensional deep sub-wavelength defect detection using $\lambda=193 \mathrm{~nm}$ optical microscopy. Opt Express 2013; 21: 26219-26226.

31 Moharam MG, Gaylord TK. Rigorous coupled-wave analysis of planar-grating diffraction. J Opt Soc Am 1981; 71: 811-818.

32 Kunz KS, Luebbers RJ. The Finite Difference Time Domain Methods for Electromagnetics. Boca Raton, FL, USA: CRC Press; 1993.

33 Pisarenco M, Maubach J, Setija I, Mattheij R. Aperiodic Fourier modal method in contrast-field formulation for simulation of scattering from finite structures. J Opt Soc Am A Opt Image Sci Vis 2010; 27: 2423-2431.

34 Silver RM, Barnes BM, Zhang NF, Zhou H, Vladár AE et al. Optimizing hybrid metrology through a consistent multi-tool parameter set and uncertainty model. In: Cain JP, editor. Metrology, Inspection, and Process Control for Microlithography XXVIII; 23-27 February 2014; San Jose, CA, USA. SPIE: Bellingham, WA, USA, 2014. p905004.

35 Honkanen M, Kettunen V, Tervo J, Turunen J. Fourier array illuminators with $100 \%$ efficiency: analytical Jones-matrix construction. J Mod Opt 2000; 47: 2351-2359.

36 Rao CR, Toutenburg H. Linear Models: Least Squares and Alternatives. Berlin: Springer; 1995

37 Bates DM, Watts DG. Nonlinear Regression Analysis and Its Applications. New York: Wiley; 1998.

38 Silver RM, Zhang NF, Barnes BM, Zhou H, Qin J et al. Nested uncertainties and hybrid metrology to improve measurement accuracy. In: Raymond C, editor. Metrology, Inspection, and Process Control for Microlithography XXV; February 27-March 3 2011; San Jose, CA, USA. SPIE: Bellingham, WA, USA, 2011. p797116.

39 Zhang NF, Silver RM, Zhou H, Barnes BM. Improving optical measurement uncertainty with combined multitool metrology using a Bayesian approach. Appl Opt 2012; 51: 6196-6206.

40 Kennedy J, Eberhart RC. A discrete binary version of the particle swarm algorithm In: 1997 IEEE International Conference on Systems, Man, and Cybernetics; 12-15 October 1997; Orlando, FL, USA. IEEE: Piscataway, NJ, USA, 1997. pp4104-4108.

41 Henn MA, Silver RM, Villarrubia JS, Zhang NF, Zhou H et al. Optimizing hybrid metrology: rigorous implementation of Bayesian and combined regression. In: Cain JP, Sanchez MI, 
editors. Metrology, Inspection, and Process Control for Microlithography XXIX; 22-26 February 2015; San Jose, CA, USA. SPIE: Bellingham, WA, USA, 2015. p94241J.

42 Endres J, Diener A, Wurm M, Bodermann B. Investigations of the influence of common approximations in scatterometry for dimensional nanometrology. Meas Sci Technol 2014; 25: 044004

43 American National Standards Institute. US Guide to the Uncertainty of Measurement. Washington: ANSI; 1997. (c) (1) (2) (2) This license allows readers to copy, distribute and transmit the Contribution as (1) transform or build upon the Contribution as long as the resulting work is then distributed under this is a similar license. Readers are not permitted to use the Contribution for commercial purposes. Please read the full license for further details at - http://creativecommons.org/ licenses/by-nc-sa/4.0/

Supplementary information for this article can be found on the Light: Science \& Applications' website: (http://www.nature.com/lsa/). 